\title{
Pediatrics and donor-derived disease transmission: The US OPTN experience
}

\author{
Michael Green $^{1}$ (D) | Shandie Covington ${ }^{2}$ | Sarah Taranto ${ }^{2}$ | Marian G. Michaels ${ }^{1}$ | \\ Cameron Wolfe $^{3}$ | Daniel R. Kaul ${ }^{4}$
}

${ }^{1}$ Division of Infectious Diseases, Departments of Pediatrics and Surgery, University of Pittsburgh School of Medicine, Children's Hospital of Pittsburgh, Pittsburgh, PA, USA

${ }^{2}$ United Network for Organ Sharing, Richmond, VA, USA

${ }^{3}$ Division of Infectious Disease, Duke University Medical Center, Durham, NC, USA

${ }^{4}$ Division of Infectious Diseases, University of Michigan Medical Center, Ann Arbor, MI, USA

\section{Correspondence}

Michael Green, Department of Pediatrics, Surgery and Clinical \& Translational Science, University of Pittsburgh School of Medicine, Pittsburgh, PA, USA.

Email: Michael.green@chp.edu

Funding information

United Network for Organ Sharing (UNOS),

Grant/Award Number: 234-2005-370011C

\begin{abstract}
PDDTE are tracked by the OPTN Ad Hoc DTAC. Specific evaluation of potential transmissions from pediatric deceased donors or the impact of donor-derived disease transmissions to pediatric organ recipients has not been previously undertaken. PDDTE reported to the DTAC between 2008 and 2013 were reviewed, characterized as proven, probable, possible, IWDT, unlikely, or excluded for both the whole event and each individual recipient. Pediatric donors and recipients were defined as being 0-17 years of age. Analysis was undertaken to characterize potential disease transmission from pediatric donors to adult or pediatric recipients and also to evaluate potential transmission from all donors to pediatric recipients. P/P cases were further analyzed. A total of 5238 pediatric deceased US donors accounted for 17456 organ transplants during the study period; 103 PDDTE reports arose from these donors (2.0\%). PDDTE were characterized as P/P (15\%), possible (13\%), IWDT (9\%), unlikely, and excluded (63\%). Disease was transmitted to 22 of 54 potentially exposed (adult and pediatric) recipients with six attributable deaths. An infectious pathogen accounted for $13 / 15$ of the P/P PDDTE associated with pediatric donors, affecting 19 of 50 potentially exposed recipients and resulting in five deaths. Four separate viral pathogens from six donors accounted for $\mathrm{P} / \mathrm{P}$ transmissions to 11 recipients with the unanticipated transmission of CMV most common. No pediatric donor transmitted HIV, $\mathrm{HBV}$, or HCV. Bacteria, fungi, and parasites accounted for three (all staphylococci), three (Zygomycetes and Histoplasma), and two (both Toxoplasma) P/P transmissions from seven donors, respectively. From the recipient side, 11/11,188 pediatric recipient deceased and living donor transplants during the study period were associated with a P/P PDDTE $(<0.1 \%)$ with infectious pathogens accounting for $9 / 11 \mathrm{P} / \mathrm{P}$ events. Infections were split among pathogen categories (bacteria 2 , viruses 3 , parasites 3 , and fungi 1 ). Reporting rates of PDDTE involving pediatric donors were very low and similar to rates from all donors, with resulting $\mathrm{P} / \mathrm{P}$ transmissions occurring in only $0.1 \%$ of exposed recipients, but transmissions were associated with six deaths. Rates of $P / P$ transmission to pediatric recipients from any donor $(<0.1 \%)$ were also very low and similar to that of all recipients. Additional studies are needed to compare the pattern and outcome of donor-derived disease transmission from and to pediatric and adult donor and recipients.
\end{abstract}


KEYWORDS

donor derived infection, infectious risk, transplantation

\section{1 | INTRODUCTION}

The UNOS is the private, non-profit organization that manages the United States' organ transplant system under the OPTN contract with HRSA. Since 2005, OPTN policy has required reporting of all unanticipated PDDTE in support of efforts to track, understand, and learn from donor-derived disease transmission events in the United States. ${ }^{1}$ Since 2008, reported PDDTE have been reviewed by the OPTN Ad Hoc DTAC. ${ }^{2}$ Since that time, the DTAC has worked to learn from this experience to enhance the transplant community's understanding of donorderived diseases and continually refine OPTN policies requiring reporting of PDDTE and communication between OPOs and transplant centers. While a growing number of publications and guidance documents have been generated through ongoing efforts to learn from this experience, neither the specific evaluation of potential transmissions from pediatric donors nor the impact of donor-derived disease transmissions on pediatric organ recipients has been previously undertaken. This study was carried out to address these issues.

\section{2 | METHODS}

This study used data from the Organ Procurement and Transplantation Network (OPTN). The OPTN data system includes data on all donors, wait-listed candidates, and transplant recipients in the United States, submitted by the members of the Organ Procurement and Transplantation Network (OPTN) and has been described elsewhere. The HRSA, US Department of Health and Human Services, provides oversight to the activities of the OPTN contractor. OPTN policy requires reporting of unexpected PDDTE to its Improving Patient Safety port (housed with $\mathrm{UNet}^{\mathrm{SM}}$ ) by either transplant hospitals or the OPOs. ${ }^{3}$ Once submitted, potential transmission events are initially screened by OPTN staff and additional details sought before undergoing formal review by the full DTAC following previously published processes and procedures. ${ }^{4}$ For each reported PDDTE, the DTAC assessed the likelihood that transmission had occurred as either proven, probable, possible, unlikely, excluded, or IWDT (typically an antimicrobial being given to prevent development of infection) for all recipients of a given donor. ${ }^{4}$ The committee's assessment for potential event included an overall assessment for a PDDTE based on the highest level (eg, proven vs possible) assigned to any of the potentially exposed recipients (eg, if one recipient experienced a proven transmission, the overall assessment would be proven regardless of the outcome for other recipients) as well as providing individual assessments for all recipients. Once all individual recipient assessments and the overall assessment were assigned for each PDDTE, this information was recorded in the OPTN DTAC database.

All cases reported to and assessed by the DTAC between 2008 and 2013 were included in this review. Potential pediatric cases were defined as involving either a donor or recipient less than 18 years of age. The DTAC database was reviewed to identify PDDTE involving a pediatric deceased donor of organs to adult or pediatric recipients as well as cases involving any age donor to pediatric recipients. All PDDTE were considered within the confines of confidential medical peer review. Because of this, the DTAC is not permitted to provide specific details for any given single potential transmission event or to provide details for any disease type where only a single report was received during the year. PDDTE classified as either proven or probable as well as those categorized as unlikely or excluded were combined for the purpose of analysis.

\section{3 | RESULTS}

An overview of donor-derived disease from pediatric donors to all recipients is summarized in Figure 1. A total of 5238 pediatric deceased donors accounted for 17456 organ transplants during the study period with 11393 going to adult recipients and 6064 to children; 103 PDDTE reports were submitted to the OPTN Improving Patient Safety Portal involving these donors (2\%). The overall evaluation by the DTAC classified 15 of these PDDTE as proven/probable for an overall rate of $0.3 \%$ of pediatric donors being associated with a proven/probable donor-derived disease transmission event. Of the remaining PDDTE, 13 were assessed to be possible, nine to be IWDT, and 66 to be unlikely/ excluded. The 15 proven/probable cases were assessed as resulting in transmission to 22 of 54 total (both pediatric and adult) exposed recipients, which represents transmission to $0.1 \%$ of all transplant recipients with pediatric organs. Infections accounted for 13/15 proven/probable PDDTE; transmission of a malignancy and a peanut allergy accounted for the remaining two proven/probable events. As summarized in Figure 1, viral infections were the most commonly transmitted class of pathogens affecting 11 recipients from six donors. Cytomegalovirus (CMV) was the most commonly transmitted virus accounting affecting four recipients from three donors. Two of the donors were teenagers while the other donor was younger. CMV would be considered an unanticipated PDDTE in situations where both the donor and recipient pretransplant serologies were negative. Despite 277 pediatric deceased donors identified as PHS increased risk during the period, no transmissions involving hepatitis ( $\mathrm{B}$ or $\mathrm{C}$ ) or HIV from a pediatric donor occurred during the study period. Bacteria, fungi, and parasites accounted for three (all staphylococci), two (Zygomycetes and Histoplasma), and two (both Toxoplasma) donor transmissions, respectively. Disease transmission was assessed as causing six deaths in two pediatric and four adult recipients. Five of the deaths were attributed to transmission of an infectious pathogen from four donors (Toxoplasma, Zygomycetes, Balamuthia, and LCMV) and one death was due to transmission of a malignancy, resulting in an attributable death rate due to a donor-derived transmission event of $0.03 \%$ of all transplant recipients of pediatric organs. 


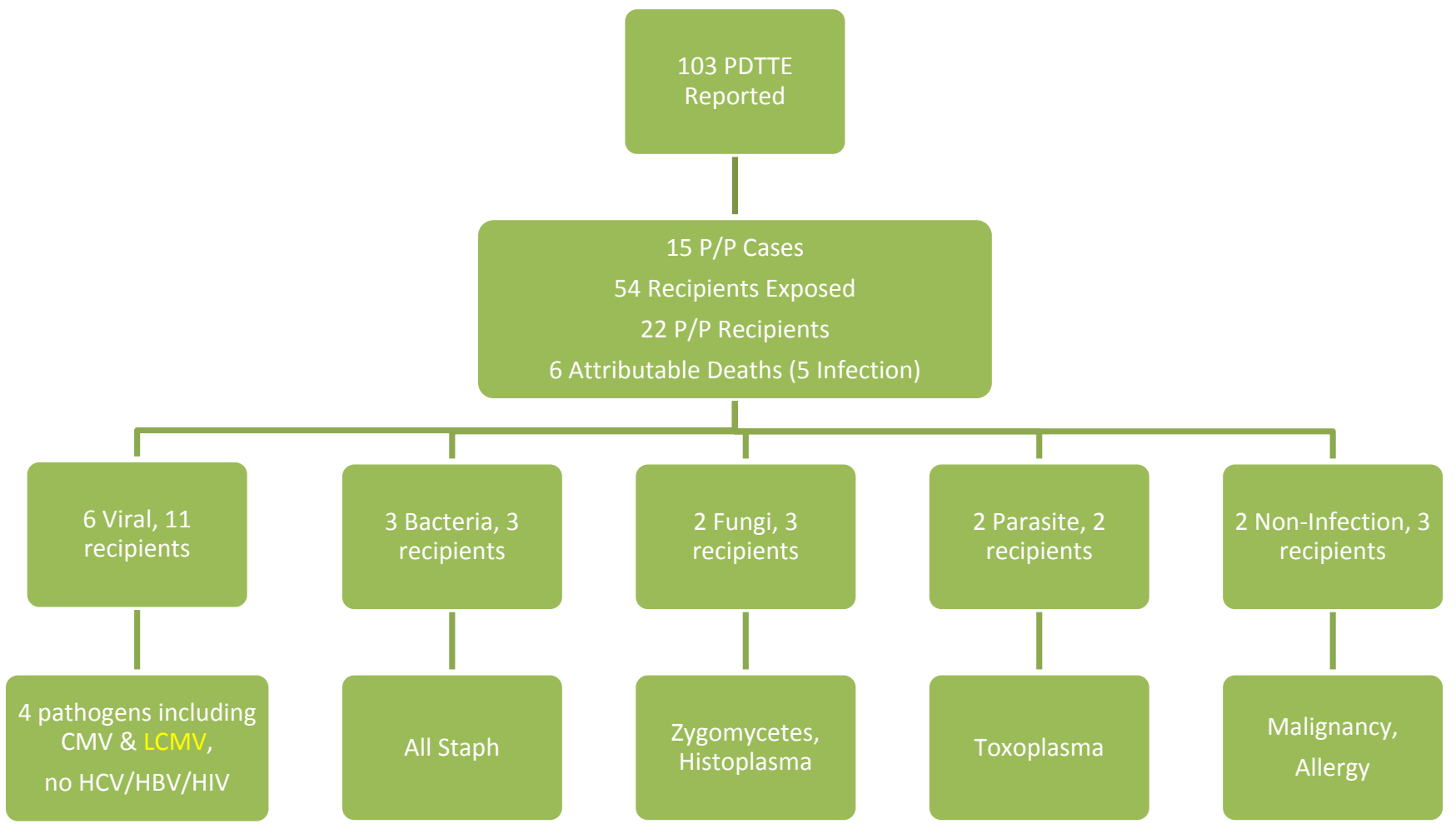

FIGURE 1 Donor-derived disease from pediatric donors: 2008-2013

A summary of donor-derived disease occurring in pediatric recipients from any donor is shown in Figure 2. A total of 11188 children received an organ from either an adult or pediatric donor during the study period. Eleven pediatric organ recipients experienced a proven/ probable PDDTE (0.1\%) with infectious pathogens accounting for 9/11 of the proven/probable transmission events. Infections were evenly split among pathogen categories (viruses 3 , bacteria 2, parasites 3 , and fungi 1 ) with eight different pathogens accounting for the nine proven/probable infectious transmissions. Pathogens specifically associated with transmission included CMV, HBV, HTLV2, Pseudomonas aeruginosa, Mycobacterium tuberculosis, Strongyloides, Toxoplasma (2 separate donors to 2 recipients), and Histoplasma. The remaining two proven/probable PDDTE were attributable to a malignancy and a peanut allergy. Proven/probable PDDTE occurred in five liver recipients (2 bacterial and 2 viral infection as well as the peanut allergy), four heart recipients (1 viral, 1 fungal, and 2 parasites), two lung recipients (1 fungal infection and 1 malignancy), and one kidney recipient (parasitic infection). Overall, proven/probable transmission events accounted for two deaths in pediatric recipients for an attributable death rate of $0.02 \%$ of all pediatric organ recipients during the study period. Of note, these two fatal transmissions were the two fatal transmission cases noted from pediatric donors.

\section{DISCUSSION}

The importance of the donor as a source of infection in organ recipients was recognized early in the history of transplantation. Seminal observations of the critical role of the donor as a major source of cytomegalovirus and Epstein-Barr virus eventually led to the recognition of a distinction between anticipated (for which one could potentially develop preventive strategies against and at a minimum provide specific consul to organ recipients and their family about the presence of risk) and unanticipated donor-derived infections. Additional observations also identified the potential for transmission of malignancies and even allergies from the donor to one of more recipients. As occurrences of high-profile donor-derived transmissions of pathogens including $\mathrm{HIV},{ }^{5} \mathrm{HCV},{ }^{5}$ rabies, ${ }^{6}$ and West Nile Virus ${ }^{7,8}$ were reported, the need for systematic tracking of potential donor-derived infections and other donor-derived diseases became increasingly recognized. In the USA, this was accomplished through mandated reporting of PDDTE to the OPTN and the creation of the OPTN Ad Hoc Disease Transmission Advisory Committee and the development of its now well-established processes and infrastructure. Additional efforts have been initiated by a number of individual countries and by the World Health Organization (Project NOTIFY). ${ }^{9}$ Taken together, these efforts share a common goal of enhancing the safety of transplant recipients through a better understanding of potential risks for disease transmission and have resulted in the enhanced awareness of the transplant community of the importance of the potential for and clinical consequences of donor-derived disease transmission, informing changes in both policy and the clinical practice of transplantation. However, while disease transmission events involving pediatric donors and pediatric recipients have been included in prior aggregate analyses, specific evaluation for unique characteristics of disease derived from pediatric donors or spectrum 


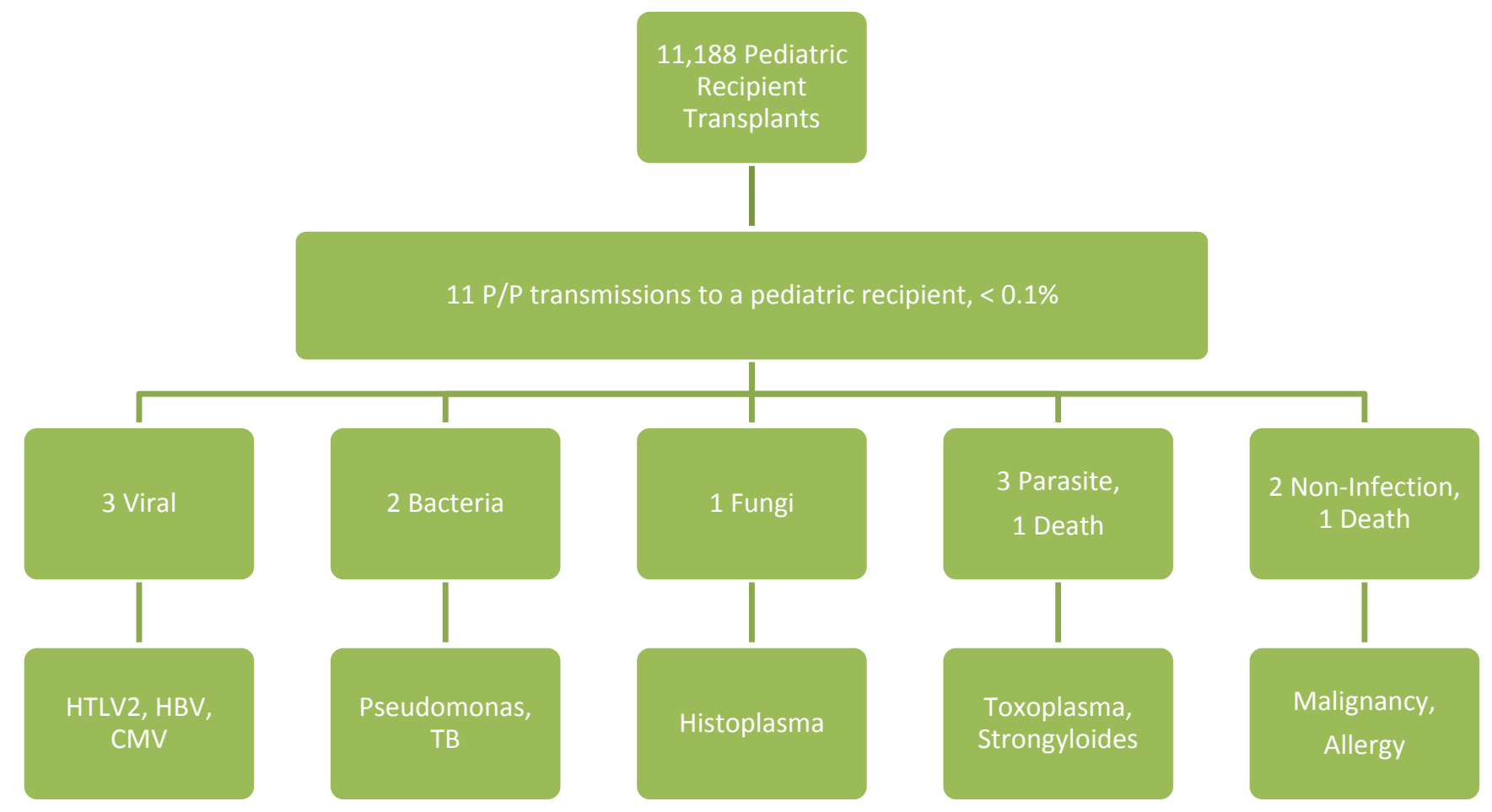

FIGURE 2 Donor-derived disease in pediatric recipients: 2008-2013

and impact of donor-derived disease on pediatric recipients have not been previously studied. Accordingly, the results of the current study are both informative and reassuring in that the incidence and outcome of donor-derived disease transmission events appear to be similar when one compares both pediatric and adult donors and recipients.

The results of the current study identified that reported rates of PDDTE involving pediatric donors were very low (2\%) with proven/ probable transmissions from pediatric donors occurring in only $0.1 \%$ of recipients (either adult or pediatric). The cumulative incidence rate of reported PDDTE was $1.9 \%$ for deceased donors (adult and pediatric) reported to the OPTN through 2013 involving donors recovered in 2008-2012 although the overall rate for all donors (deceased and living) was $1.1 \% .{ }^{4}$ The rate of proven/probable transmission during this time period was $0.4 \%$ and $0.2 \%$ from deceased donors (adult and pediatric) and all donors (living and deceased), respectively. ${ }^{4}$ As children are rarely if ever used as living donors, comparison of both the rate of PDDTE reporting and that of proven/probable transmission between pediatric and all deceased donors would seem most appropriate. Based upon these comparisons, the current results suggest that rates of both reporting a potential PDDTE and of a proven/probable transmission were similar for pediatric and adult deceased donors during this time period.

Five proven/probable disease transmission events from a pediatric donor were assessed as causing six deaths ( 2 in pediatric and 4 in adult recipients) in this study for an attributable mortality rate of $0.03 \%$ of all recipients of pediatric organs. Five of the six deaths were associated with the transmission of an infectious pathogen, and one was due to transmission of a malignancy. Two pediatric recipients were assessed as dying secondary to a donor-derived transmission (both from pediatric donors) for an attributable mortality rate of $0.02 \%$. In comparison, analysis of proven/probable disease transmission from all deceased donors (adult and pediatric) during this time period identified 39 deaths affecting the 110402 recipients transplanted for an attributable mortality rate of $0.04 \%$. Accordingly, rates of attributable mortality due to proven/probable disease transmission appear to be similar in both the adult and pediatric donor and recipient populations.

Infectious pathogens accounted for 13 of 15 (87\%) of proven/ probable donor-derived transmission events from pediatric donors during this study period. Viral pathogens accounted $46 \%$ of these transmissions, but important transmission events also occurred due to bacteria, fungi, and parasites. In comparison, in a cohort including all donors (but largely adult donors), viral infections accounted for 42 of 219 (19\%) of transmission with 19 hepatitis C or hepatitis B transmissions, pathogens not transmitted from our pediatric cohort despite the fact that it included 277 donors identified as PHS increased risk. Further malignancies and non-infectious events accounted for the largest percentage of adult donor transmission events (30\%) and the largest number of fatal donor transmissions, but were very uncommon in the pediatric population. ${ }^{10}$

Limitations include the reliance of all recipient centers and OPOs to report suspected donor-derived diseases for review through what is a passive reporting system. In addition, the data for the DTAC members to review are limited to deidentified information with follow-up generally confined to 45 days after the event. Accordingly, some cases may have been either under- or overclassified.

In summary, reported rates of PDDTE involving pediatric donors were very low and similar to rates from all donors, with resulting 
$\mathrm{P} / \mathrm{P}$ transmissions occurring in only $0.1 \%$ of exposed recipients, but transmissions were associated with six deaths. Rates of P/P transmission to pediatric recipients from any donor $(<0.1 \%)$ were also very low and similar to that of all recipients. Additional studies are needed to compare the pattern and outcome of donor-derived disease transmission from and to pediatric and adult donor and recipients.

\section{ACKNOWLEDGMENTS}

This work was conducted under the auspices of the United Network for Organ Sharing (UNOS), contractor for OPTN, under Contract 234-2005-370011C (US Department of Health and Human Services, Health Resources and Services Administration, Healthcare Systems Bureau, Division of Transplantation). The data reported here have been supplied by the United Network for Organ Sharing (UNOS) as the contractor for the Organ Procurement and Transplantation Network (OPTN). The interpretation and reporting of these data are the responsibility of the author(s) and in no way should be seen as an official policy of or interpretation by the OPTN or the U.S. Government.

\section{ORCID}

Michael Green (iD http://orcid.org/0000-0002-6011-2894

\section{REFERENCES}

1. Ison MG, Hager J, Blumberg E, et al. Donor-derived disease transmission events in the United States: data reviewed by the OPTN/ UNOS Disease Transmission Advisory Committee. Am J Transplant. 2009;9:1929-1935.
2. Ad Hoc Disease Transmission Advisory Committee. http://optn. transplant.hrsa.gov/members/committeesDetail.asp?/D=95. Accessed September 19, 2014.

3. OPTN Policy 15.4. http://optn.transplant.hrsa.gov/policiesAndBylaws/policies.asp. Accessed September 19, 2014.

4. Green M, Covington S, Taranto S, et al. Donor-derived transmission events in 2013: a report of the Organ Procurement Transplant Network Ad Hoc Disease Transmission Advisory Committee. Transplantation 2015;99:282-287.

5. Ison MG, Llata E, Conover CS, et al. Transmission of human immune deficiency virus and hepatitis $C$ virus from an organ donor to four transplant recipients. Am J Transplant. 2011;11:1218-1225.

6. Srinivasan A, Burton EC, Kuehnert MJ, et al. Transmission of rabies virus from an organ donor to four transplant recipients. N Engl J Med. 2005;352:1103-1111.

7. Iwato M, Jernigan DB, Guasch A, et al. Transmission of West Nile Virus from an organ donor to four transplant recipients. N Engl I Med. 2003;348:2196-2203.

8. Centers for Disease Control and Prevention (CDC). West Nile virus infections in organ transplant recipients-New York and Pennsylvania. MMWR Morb Mortal Wkly Rep. 2005;54:1021-1023.

9. Ison M, Strong D, Fehily E, Chatzixiros A, Costa LN. Project NOTIFY: a global database of serious adverse events and reactions in organs, tissues and cells. Abstract 787. Am J Transplant. 2013;13 (suppl 5):268

10. Kaul D, Tlusty S, Wilk A, Wolfe C. A Decade of Donor-Derived Disease: A Report of the OPTN Ad Hoc Disease Transmission Advisory Committee. Boston, MA: American Transplant Congress; 2016.

How to cite this article: Green M, Covington S, Taranto S, Michaels MG, Wolfe C, Kaul DR. Pediatrics and donor-derived disease transmission: The US OPTN experience. Pediatr Transplantation. 2018;22:e13115. https://doi.org/10.1111/ petr.13115 